\title{
Image-guided selection of Gd@C-dots as sensitizers to improve radiotherapy of non-small cell lung cancer
}

Xiaofen $\mathrm{Ma}^{1,2 \dagger}$, Chaebin Lee ${ }^{3 \dagger}$, Tao Zhang ${ }^{2 \dagger}$, Jinghua Cai ${ }^{2}$, Hui Wang ${ }^{2}$, Fangchao Jiang ${ }^{3}$, Zhanhong Wu ${ }^{2}$, Jin $\mathrm{Xie}^{3^{*}}$, Guihua Jiang ${ }^{1^{*}}$ and Zibo $\mathrm{Li}^{2^{*}}$ (D)

\begin{abstract}
Background: Recently, gadolinium-intercalated carbon dots (Gd@C-dots) have demonstrated potential advantages over traditional high-Z nanoparticles (HZNPS) as radiosensitizers due to their high stability, minimal metal leakage, and remarkable efficacy.

Results: In this work, two Gd@C-dots formulations were fabricated which bore carboxylic acid (CA-Gd@C-dots) or amino group (pPD-Gd@C-dots), respectively, on the carbon shell. While it is critical to develop innovative nanomateirals for cancer therapy, determining their tumor accumulation and retention is equally important. Therefore, in vivo positron emission tomography (PET) was performed, which found that ${ }^{64} \mathrm{Cu}$-labeled pPD-Gd@C-dots demonstrated significantly improved tumor retention (up to 48 h post injection) compared with CA-Gd@C-dots. Indeed, cell uptake of ${ }^{64} \mathrm{Cu}$-pPD-Gd@C-dots reached close to 60\% of total dose compared with 5\% of ${ }^{64} \mathrm{Cu}$-CA-Gd@C-dots. pPD-Gd@C-dots was therefore further evaluated as a new radiosensitizer for non-small cell lung cancer treatment. While single dose radiation plus intratumorally injected pPD-Gd@C-dots did lead to improved tumor suppression, the inhibition effect was further improved with two doses of radiation. The persistent retention of pPD-Gd@C-dots in tumor region eliminates the need of reinjecting radiosensitizer for the second radiation.
\end{abstract}

Conclusions: PET offers a simple and straightforward way to study nanoparticle retention in vivo, and the selected pPD-Gd@C-dots hold great potential as an effective radiosensitizer.

Keywords: Gadolinium, Carbon dots, Radiosensitizers, PET imaging, Non-small cell lung cancer, Radiation therapy

*Correspondence: jinxie@uga.edu; jiangguihua1970@163.com; ziboli@med. unc.edu

${ }^{+}$Xiaofen Ma, Chaebin Lee and Tao Zhang contributed equally to the research.

${ }^{1}$ Department of Nuclear Medicine, Guangdong Second Provincial General Hospital, 466 Xingang Middle Road, Haizhu District, Guangdong Province 510317 Guangzhou City, People's Republic of China

2 Department of Radiology, Biomedical Research Imaging Center, and Lineberger Comprehensive Cancer Center, University of North Carolina at Chapel Hill, 125 Mason Farm Road, Chapel Hill, NC 27599, USA ${ }^{3}$ Department of Chemistry, University of Georgia, 140 Cedar Street, Athens, GA 30602, USA

\section{Introduction}

Radiation therapy (RT) is one of the most widely used cancer treatment modalities [1-3]. However, significant side effects are often observed due to RT-induced damage to surrounding normal tissues [4-7]. Selectively increasing energy deposition at the tumor region could potentially improve tumor treatment efficacy and reduce the associated side effects. Over the last decade, various nanomedicines have been developed and tested as radiosensitizing agents, among which high- $Z$ nanoparticles (HZNPs) have attracted extensive attention [8-13]. HZNPs afford large cross-section for high energy photons, thus producing more photo- or Auger-electrons original author(s) and the source, provide a link to the Creative Commons licence, and indicate if changes were made. The images or other third party material in this article are included in the article's Creative Commons licence, unless indicated otherwise in a credit line to the material. If material is not included in the article's Creative Commons licence and your intended use is not permitted by statutory regulation or exceeds the permitted use, you will need to obtain permission directly from the copyright holder. To view a copy of this licence, visit http://creativecommons.org/licenses/by/4.0/. The Creative Commons Public Domain Dedication waiver (http://creativeco mmons.org/publicdomain/zero/1.0/) applies to the data made available in this article, unless otherwise stated in a credit line to the data. 
(the effect of which is varied by $\mathrm{Z}^{3}$ or $\mathrm{Z}^{4}$ ) under radiation that damage cancer cells. Such radiosensitizing effects were observed in HZNPs made of gold, silver, bismuth, and gadolinium [14-19]. Excitingly, NBTXR3, a hafnium oxide formulation, has demonstrated clinical benefits and received regulatory approval in the EU for enhancing RT $[20,21]$.

Despite promising results, many conventional HZNPs are associated with issues such as a large particle size, uncontrolled metal leakage, and heavy-metal toxicity [22-25]. Moreover, dose modifying factors of nanoparticles are hard to predict without a costly and lengthy animal experiments $[8,26,27]$. In this report, we tested two ultrasmall Gd-encapsulated carbon dots (Gd@C-dots), CA-Gd@C-dots and pPD-Gd@C-dots, which bear positive and neutral surface charges, respectively. We speculated that in addition to particle size, surface properties could impact the in vitro and in vivo behaviors of Gd@ Cdots and in turn affect their role as a radiosensitizer. Positron emission tomography (PET) is a noninvasive imaging technology that allows us to track radiolabeled ligands and materials quantitatively and repetitively. In this report, we labeled both CA-Gd@C-dots and pPDGd@C-dots with ${ }^{64} \mathrm{Cu}$ and investigated the nanoparticles' uptake by cancer cells and retention in tumors using PET. We exploit this quantitative molecular imaging method to monitor nanoparticle clearance after injection and screen for a better formulation. Overall, the research not only evaluated new radiosensitizers based on Gd@Cdots, but also demonstrated that PET could be used as a tool to examine clearance of intratumorally injected nanoparticles so as to facilitate nanomedicine research.

\section{Materials and methods}

\section{Materials}

All chemicals and solvents were obtained from commercial sources and used without further purification. p-Phenylenediamine (pPD, Sigma Aldrich, Cat\# 78429), Citric acid (CA, Sigma Aldrich, Cat\# 251275), Gadolinium nitrate hexahydrate $\left(\mathrm{Gd}\left(\mathrm{NO}_{3}\right)_{3} \cdot 6 \mathrm{H}_{2} \mathrm{O}\right.$, Sigma Aldrich, Cat\# 211591), Ethanol (KOPTEC, Cat\# 19J14D), Dialysis membrane (Spectrum, $[\mathrm{MWCO}]=100-500$ ), Milli-Q $\quad \mathrm{H}_{2} \mathrm{O}, \quad 3-(4,5-$ Dimethylthiazolyl-2)-2,5-diphenyltetrazolium bromide (MTT) (Sigma Aldrich, Cat\# M2128).

All experimental procedures involving animals were executed accordance with animal protocol approved by University of North Carolina at Chapel Hill Institutional Animal Care and Use Committee.

\section{The synthesis of CA-Gd@C-dots and pPD-Gd@C-dots}

Gd@C-dots were synthesized by hydrothermal reaction. Briefly, $1.33 \mathrm{mmol}$ of $\mathrm{Gd}\left(\mathrm{NO}_{3}\right)_{3} \cdot 6 \mathrm{H}_{2} \mathrm{O}$ and $1.48 \mathrm{mmol}$ of carbon precursor ( $\mathrm{pPD}$ or $\mathrm{CA}$ ) was dissolved in $60 \mathrm{~mL}$ of either ethanol or Milli-Q $\mathrm{H}_{2} \mathrm{O}$ by vortex mixer, and the solution was transferred into a $100 \mathrm{~mL}$ poly(tetrafluoroethylene)-lined autoclave. The autoclave was kept in an oven, and the temperature was increased at a rate of $3{ }^{\circ} \mathrm{C} / \mathrm{min}$ to $180{ }^{\circ} \mathrm{C}$. After $12 \mathrm{~h}$, the autoclave was cooled down to room temperature. The raw products were washed three times with water, followed by dialysis (MWCO 500) that removed carbon byproducts and free gadolinium ions. The final products were lyophilized and stored at $-80^{\circ} \mathrm{C}$.

\section{Characterization of CA-Gd@C-dots and pPD-Gd@C-dots}

Transmission electron microscopy (TEM) images were acquired on a FEI TECNAI 20 transmission electron microscope operated at $200 \mathrm{kV}$. The zeta potential of the nanoparticles was measured by a Malvern Zetasizer Nano ZS system. Energy-dispersive X-ray spectroscopy (EDS) and element mapping were performed on a FEI Inspect F FEG-SEM system equipped with EDZX EDS. Inductively coupled plasma mass spectrometry (ICP-MS) was used to quantify Gd content in the samples.

\section{Preparation of DOTA conjugated Gd@C-dots}

DOTA-CA-Gd@C-dots was prepared according to the reference procedure [28]. Briefly, CA-Gd@C-dots was activated by EDC and sNHS at pH 5.5 for 30 min with a CA-Gd@C-dots:EDC:sNHS molar ratio of 10:5:4. The activated CA-NHS-Gd@C-dots) $(0.92 \mu \mathrm{mol}$, calculated on the basis of sNHS, $300 \mu \mathrm{L}$ ) were then added into a 200 $\mu \mathrm{L}$ DOTA-Amine (MW 766.4, $500 \mu \mathrm{g}, 0.65 \mu \mathrm{mol}$ ) solution at a pH of 8.5. The mixture was incubated at $4{ }^{\circ} \mathrm{C}$ for $12 \mathrm{~h}$, and the unreacted materials were removed using a $3 \mathrm{k}, 4 \mathrm{~mL}$ micro filter unit (dilute the reaction solution to $4 \mathrm{~mL}$ by adding deionized water; centrifuge at $3500 \mathrm{rpm}$ for $15 \mathrm{~min}$; and disperse using pipette. Repeat this procedure three times until $250 \mu \mathrm{L}$ of particle was left on the filter).

DOTA-NHS $(100 \mu \mathrm{g}, 0.13 \mu \mathrm{mol})$ was added into 200 $\mu \mathrm{L}$ pPD-Gd@C-dots NPs $(2.2 \mu \mathrm{mol}$, calculated on the basis of $-\mathrm{NH}_{2}$ group) solution at a $\mathrm{pH}$ of 8.5 . The mixture was incubated at $4{ }^{\circ} \mathrm{C}$ for $12 \mathrm{~h}$, and the unreacted materials were removed using a $10 \mathrm{k}, 4 \mathrm{~mL}$ micro filter unit (dilute the reaction solution to $4 \mathrm{~mL}$ by adding deionized water; centrifuge at $3500 \mathrm{rpm}$ for $15 \mathrm{~min}$ and disperse using pipette. Repeat this three times until $100 \mu \mathrm{L}$ of particle was left on the filter) to obtain DOTA-pPDGd@C-dots. Similarly, NOTA-NCS $(50 \mu \mathrm{g}, 0.09 \mu \mathrm{mol})$ was added into $200 \mu \mathrm{L}$ pPD-Gd@C-dots $(2.2 \mu \mathrm{mol}$, calculated on the basis of $-\mathrm{NH}_{2}$ group) solution at a $\mathrm{pH}$ of 9.5. The mixture was incubated at $4{ }^{\circ} \mathrm{C}$ for $12 \mathrm{~h}$, and the unreacted materials were removed using a $10 \mathrm{k}, 4 \mathrm{~mL}$ micro filter unit (dilute the reaction solution to $4 \mathrm{~mL}$ 
by adding deionized water; centrifuge at $3500 \mathrm{rpm}$ for $15 \mathrm{~min}$ and disperse using pipette. Repeat this three times until $100 \mu \mathrm{L}$ of particle was left on the filter) to obtain NOTA-pPD-Gd@C-dots.

\section{Radiochemistry}

${ }^{64} \mathrm{Cu}$-DOTA-CA-Gd@C-dots, ${ }^{64} \mathrm{Cu}-\mathrm{DOTA}-\mathrm{pPD}-\mathrm{Gd} @ \mathrm{C}-$ dots and ${ }^{64} \mathrm{Cu}-\mathrm{NOTA}-\mathrm{pPD}-\mathrm{Gd} @ \mathrm{C}$-dots $(50 \mu \mathrm{L}$, respectively) were prepared by the addition of ${ }^{64} \mathrm{Cu}(2 \mathrm{mCi}$, respectively) and chelator modified particles in $0.25 \mathrm{M}$ ammonium acetate (pH 5.5) buffer, and the mixture was incubated for $30 \mathrm{~min}$ at $40{ }^{\circ} \mathrm{C} .{ }^{64} \mathrm{Cu}$ labeled Gd@C-dots were then purified using a micro filter unit with deionized water as the washing solution. The radioactive fractions containing ${ }^{64} \mathrm{Cu}$-DOTA-Gd@C-dots were collected for further in vitro and in vivo experiments.

\section{Cells and animals}

H1299 is a human NSCLC cell line. H1299 cells were grown in RPMI 1640 medium supplemented with $10 \%$ FBS and maintained in a humidified, $5 \%$ carbon dioxide
$\left(\mathrm{CO}_{2}\right)$ atmosphere at $37{ }^{\circ} \mathrm{C}$ until sufficient cells were available.

To generate H1299 tumor model, the female nude mice were injected subcutaneously with $\mathrm{H} 1299$ cells at a concentration of $2 \times 10^{6}$ cells in $100 \mu \mathrm{L}$ of phosphate buffered saline in the thigh. Tumors were established within 3-4 week after inoculation (the tumor volume, about 100 $\mathrm{mm}^{3}$ ). The mice were subjected to small-animal PET and therapy studies.

\section{In vitro toxicity study}

The cell viability was studied with $\mathrm{H} 1299$ cells line following to the standard MTT assays. The H1299 cells were seeded on clear 96 -well plates (8000 cells per well) in $100 \mu \mathrm{L}$ cell culture media. After $24 \mathrm{~h}, 100 \mu \mathrm{L}$ of Gd@Cdots (either CA or pPD) containing cell culture media in range of concentration $(0-400 \mu \mathrm{g} / \mathrm{mL}, \mathrm{Gd})$ were added to each well and incubated at $37{ }^{\circ} \mathrm{C}$ with the cells for $24 \mathrm{~h}$. Then, $10 \mu \mathrm{L}$ of MTT solution $(10 \mathrm{mg} / \mathrm{mL})$ was added to each well and stored in the incubator for another $4 \mathrm{~h}$. All solution was aspirated, and $100 \mu \mathrm{L}$ of DMSO was added
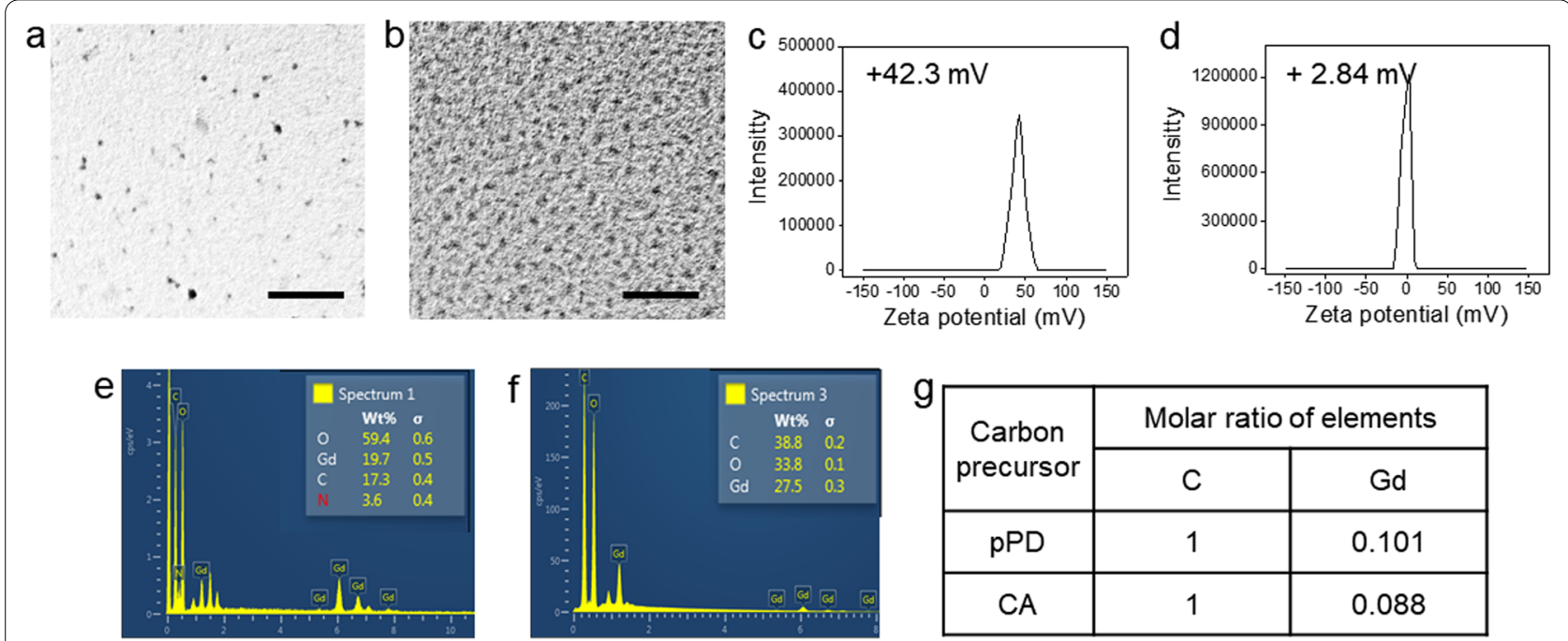

\begin{tabular}{|c|c|c|}
\hline \multirow{2}{*}{$\begin{array}{c}\text { Carbon } \\
\text { precursor }\end{array}$} & \multicolumn{2}{|c|}{ Molar ratio of elements } \\
\cline { 2 - 3 } & $\mathrm{C}$ & $\mathrm{Gd}$ \\
\hline $\mathrm{pPD}$ & 1 & 0.101 \\
\hline $\mathrm{CA}$ & 1 & 0.088 \\
\hline
\end{tabular}
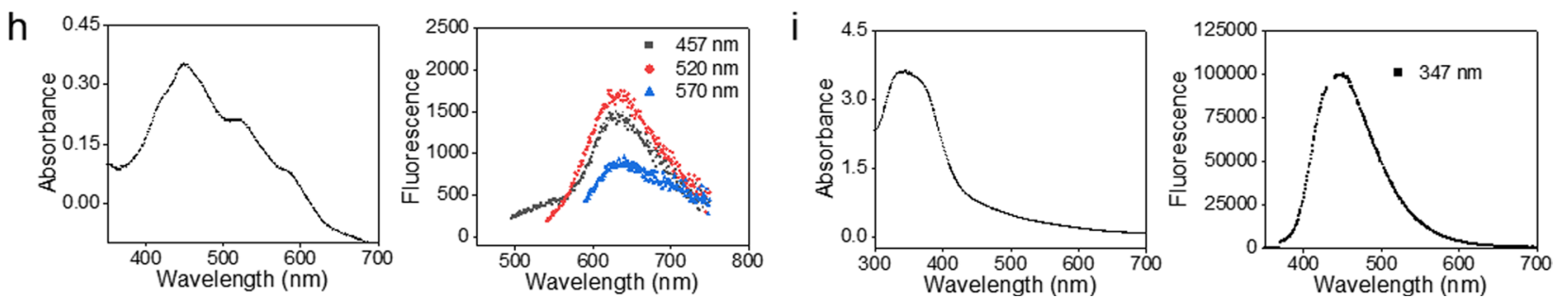

Fig. 1 Synthesis and characterizations of Gd@C-dots. a, b Representative TEM images of pPD-Gd@C-dots (a) and CA-Gd@C-dots (b).The scale bars are 100 nm. c, d DLS analysis. pPD-Gd@C-dots (c) and CA-Gd@C-dots (d) bear positive (+ $42.3 \mathrm{mV})$ and almost neutral (+ 2.84) surfaces, respectively. e, f EDS elemental analysis of pPD-Gd@C-dots (e) and CA-Gd@C-dots (f).gTable showing compositions of pPD-Gd@C-dots and CA-Gd@C-dots, based on EDS results. h Uv-vis absorption (left) and fluorescence (right) spectra of pPD-Gd@C-dots. pPD-Gd@C-dots have absorbance peak at $457 \mathrm{~nm}, 520 \mathrm{~nm}$, and $570 \mathrm{~nm}$, and an emission peak at $630 \mathrm{~nm}$ (excited at 457, 520, or $570 \mathrm{~nm}$ ). (i) Uv-vis absorption (left) and fluorescence (right) spectra of CA-Gd@C-dots. CA-Gd@C-dots have an absorbance peak at 347 nm and emission peak at 449 nm (excited at 347 nm) 


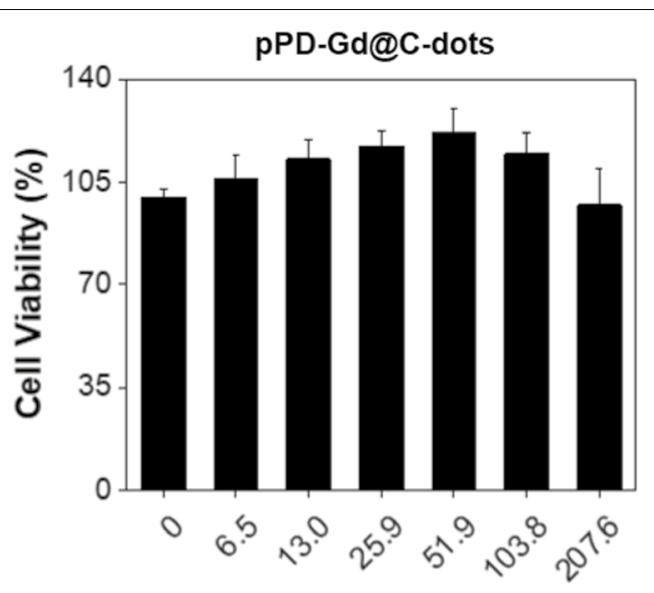

Conc. $(\mu \mathrm{g} / \mathrm{mL})$

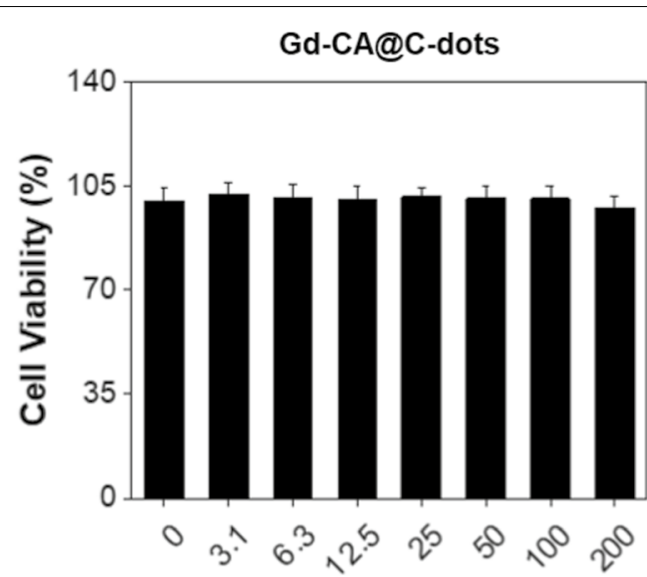

Conc. $(\mu \mathrm{g} / \mathrm{mL})$

Fig. 2 Cytotoxicity of pPD-Gd@C-dots and CA-Gd@C-dots, measured by MTT assay at 24 h on H1299 cells. No significant toxicity was observed up to $200 \mu \mathrm{g} \mathrm{Gd} / \mathrm{mL}$

to each well. The plate was shaked for $10 \mathrm{~min}$, and the absorbance at $570 \mathrm{~nm}$ was read by BioTek Synergy MX multi-mode microplate reader.

\section{ATP assay for cytotoxicity evaluation with $\mathrm{H} 1299$ cells}

The cell viability was evaluated on H1299 cells using standard 1Step ATP assay (PerkinElmer, ATPlite 1step Luminescence Assay Cat\#6016736). The H1299 cells were seeded on opaque 96-well plates (8000 cells per well) in $100 \mu \mathrm{L}$ cell culture media. After $24 \mathrm{~h}, 100 \mu \mathrm{L}$ of Gd@C-dots (either CA or pPD) at a concentrations $(0-400 \mu \mathrm{g} / \mathrm{mL}, \mathrm{Gd})$ were added into each well and incubated at $37{ }^{\circ} \mathrm{C}$ with the cells for $24 \mathrm{~h}$. Then, cell medium were removed and cells were rinsed with PBS three times before $100 \mu \mathrm{L}$ of ATP assay buffer was added to each well (Additional file 1: Fig S2). The plate was kept in dark for $10 \mathrm{~min}$ and the overall luminescence was read by a BioTek Synergy MX multi-mode microplate reader.

\section{Cell uptake}

For cell uptake assay, H1299 cells were seeded into a 24-well plate at a density of $1 \times 10^{5}$ cells per well and incubated with $\sim \mu \mathrm{Ci}{ }^{64} \mathrm{Cu}-\mathrm{DOTA}-\mathrm{CA}-\mathrm{Gd} @ \mathrm{C}$-dots, ${ }^{64} \mathrm{Cu}$-DOTA-pPD-Gd@C-dots and ${ }^{64} \mathrm{Cu}-\mathrm{NOTA}-\mathrm{pPD}-$ Gd@C-dots at $37{ }^{\circ} \mathrm{C}$. After incubation for 5, 15, 30, 60, 120 , and $240 \mathrm{~min}$, the tumor cells were washed twice with cold PBS and harvested by adding $250 \mu \mathrm{L} 2 \mathrm{~N} \mathrm{NaOH}$. The total cell lysate at each time point was collected and the radioactivity was measured in a gamma counter. The cell uptake was expressed as the percentage of the added dose after decay correction. Each data point is an average of quadruplicates.

\section{In vivo small-animal PET studies}

PET scans and image analysis were performed as previously reported [29, 30]. For small-animal PET imaging, H1299 tumor model mice were intratumorally injected with $2.15 \mathrm{MBq}$ of ${ }^{64} \mathrm{Cu}-D O T A-C A-G d @ C-d o t s$ and $0.89 \mathrm{MBq}{ }^{64} \mathrm{Cu}-D O T A-p P D-G d @ C$-dots. Serial imaging $(1,4,24$ and $48 \mathrm{~h}$ after injection; static scan duration 10, 10, 10, and $30 \mathrm{~min}$, respectively) was performed using a small-animal PET scanner (Super Argus). Next, the images were reconstructed using a 2-dimensional ordered-subsets expectation maximization algorithm. Next, for each image, regions of interest (ROIs) were drawn over the tumor, major tissue, and organ using amide software. Assuming a tissue density of $1 \mathrm{~g} / \mathrm{mL}$, the means were converted to counts per gram per minute. Dividing counts per gram per minute by injected dose

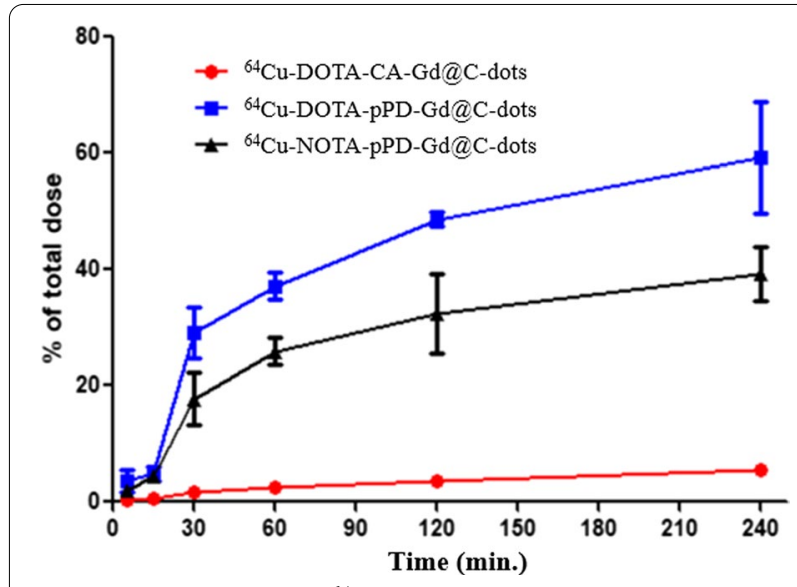

Fig. 3 Cell uptake assay of ${ }^{64} \mathrm{Cu}$ labeled Gd@C-dots in H1299 cells 
gave the image ROI-derived percentage injected dose per gram tissue $(\% \mathrm{ID} / \mathrm{g})$ values.

\section{$\mathrm{X}$-ray radiotherapy studies}

H1299 subcutaneous mouse model was used for the therapy study. Twelve mice with tumors of approximately $100 \mathrm{~mm}^{3}$ were randomly divided into four groups. For the treatment group, the mice were first intratumorally injected with $100 \mu \mathrm{g}$ pPD-Gd@C-dots $(4 \mathrm{mg} / \mathrm{mL}, 25 \mu \mathrm{L})$. After $1 \mathrm{~h}, \mathrm{X}$-ray irradiation (6 Gy) was applied to tumors, with the rest of the animal body covered by 6 -layer lead. The three control groups received: (1) control; (2) $100 \mu \mathrm{g}$ pPD-Gd@C-dots alone; (3) X-Ray irradiation (6 Gy) only. After the treatment, to monitor tumor growth, the tumor sizes and body weights were measured at different time points. Tumor sizes were measured by a caliper and calculated using this formula: size $\left(\mathrm{mm}^{3}\right)=$ length $(\mathrm{mm}) \times$ width $(\mathrm{mm})^{2} / 2$.

In addition, we used $12 \mathrm{H} 1299$ mice for another treatment study. For the treatment group, the mice were first intratumorally injected with $100 \mu \mathrm{g}$ pPD-Gd@C-dots $(4 \mathrm{mg} / \mathrm{mL}, 25 \mu \mathrm{L})$. After $1 \mathrm{~h}$, X-Ray irradiation (6 Gy) was applied to tumors, with the rest of the animal body covered by 6-layer lead. Three days later, the same treatment was given to these mice. The three control groups received: (1) Control; (2) $100 \mu \mathrm{g}$ pPD-Gd@C-dots alone; (3) X-ray irradiation (6 Gy) only (twice). After the treatment, the tumor sizes and body weights were measured at different time points in the same way.

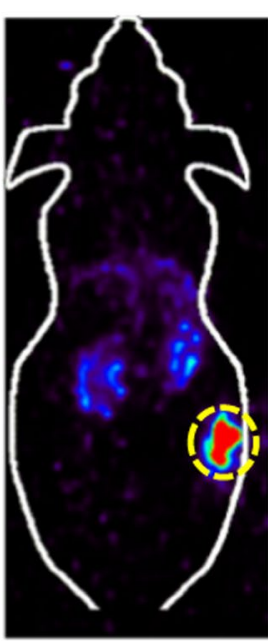

1h

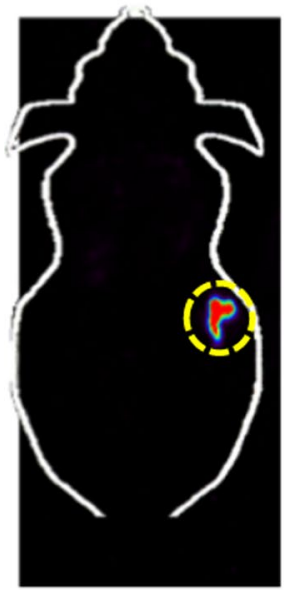

$1 \mathrm{~h}$

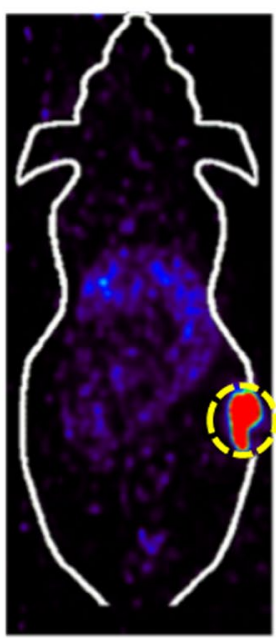

$4 \mathrm{~h}$

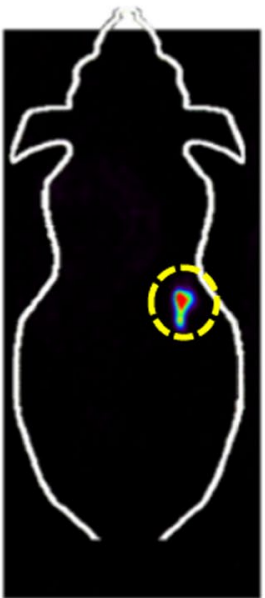

$4 \mathrm{~h}$

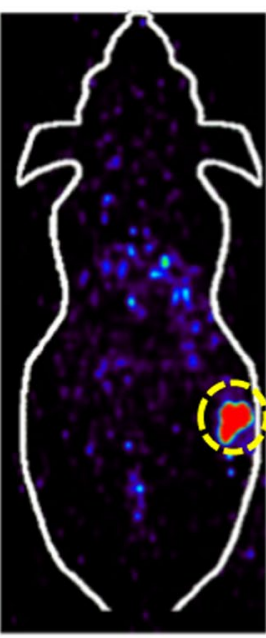

$24 \mathrm{~h}$

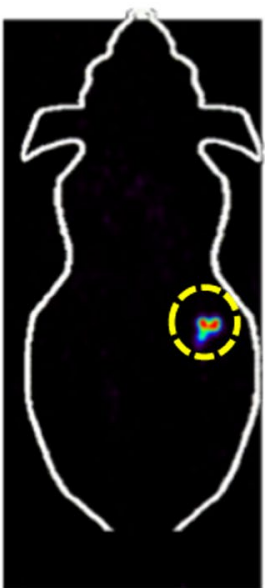

$24 \mathrm{~h}$

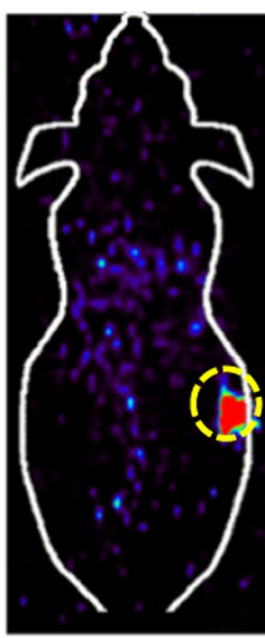

$48 \mathrm{~h}$

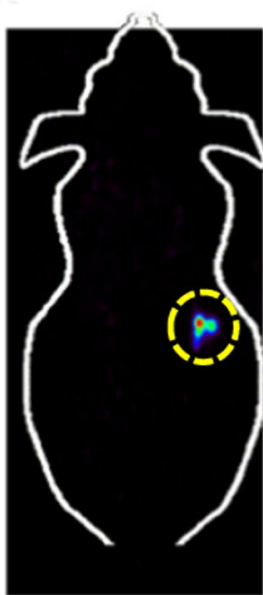

$48 \mathrm{~h}$

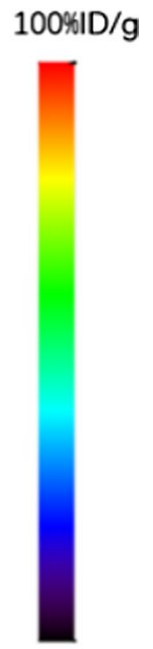

$2000 \% 1 D / g$

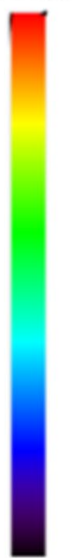

Fig. 4 Representative coronal small-animal PET images of mice bearing H1 299 xenografts at 1, 4, 24, and 48 h after Intratumoral injection of $0.89 \mathrm{MBq}$ of ${ }^{64} \mathrm{Cu}$-DOTA-pPD-Gd@C-dots with scope bar 100\%lD/g(top) and 2000\%ID/g (bottom) 

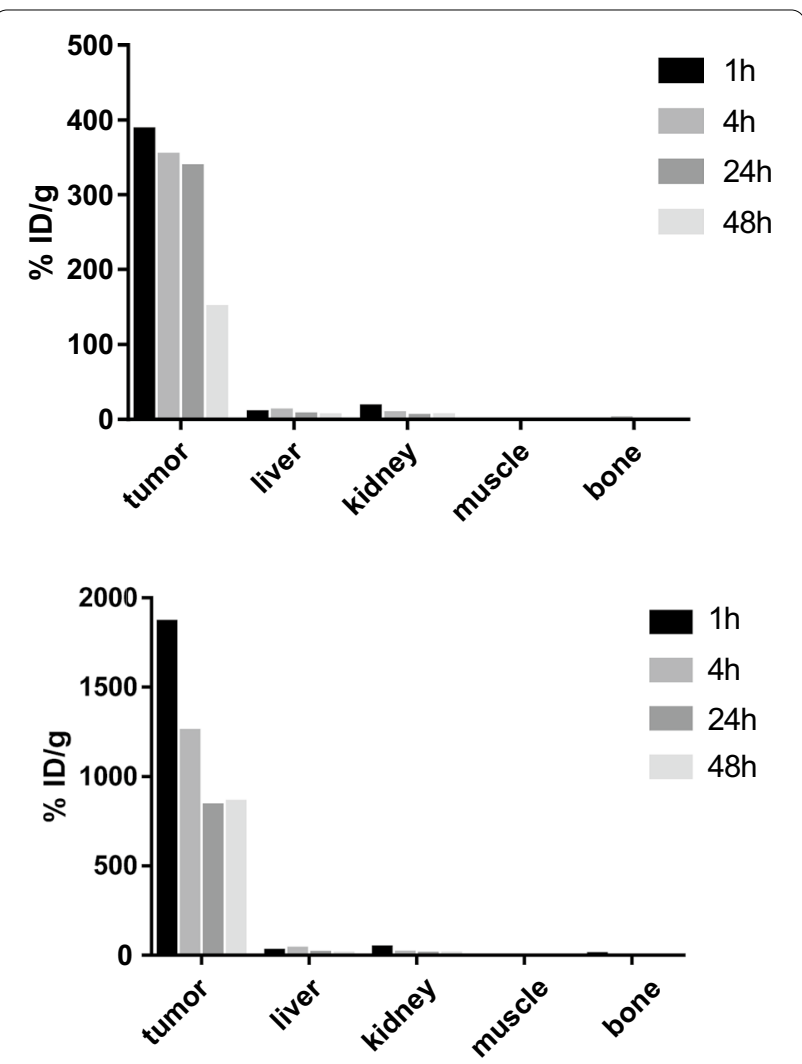

Fig. 5 Comparison of tumor, liver, kidney, muscle and bone uptake of ${ }^{64} \mathrm{Cu}$-DOTA-pPD-Gd@C-dots at different time points (1, 4, 24, and $48 \mathrm{~h}$ ) after intratumoral injection

\section{Results and discussion}

\section{Preparation and characterizations of Gd@C-dots}

Gd@C-dots were synthesized through hydrothermal reaction using p-phenylenediamine or citric acid as carbon precursor and $\mathrm{Gd}\left(\mathrm{NO}_{3}\right)_{3}$ as gadolinium precursor [31-33]. After reaction, the raw products were purified by dialysis to remove unreacted precursors and surfacebound metals. Both types of nanoparticles can be stably dispersed in water. While pPD-Gd@C-dots were red in color, CA-Gd@C-dots appeared brown (Additional file 1: Fig. S1).

Transmission electron microscopy (TEM) found that both nanoparticles have a diameter of $\sim 3 \mathrm{~nm}$ (Fig. 1a, b). Zeta potential analysis found that pPD-Gd@C-dots are positively charged on the surface $(+42.3 \mathrm{mV}$, Fig. $1 \mathrm{c})$, while CA-Gd@C-dots are virtually neutral $(+2.84 \mathrm{mV}$, Fig. 1d). The positive surface charge of the former is attributed to surface amine groups inherited from the pPD precursor. Energy dispersive spectroscopy (EDS) found that the C-to-Gd molar ratio was similar, which is 1:0.1 for pPD-Gd@C-dots and 1:0.09 for CA-Gd@C-dots (Fig. 1e-g).
The optical properties are also different between the two nanoparticles. Specifically, pPD-Gd@C-dots showed an absorbance peak at $457 \mathrm{~nm}$, and two shoulders at 520 and $570 \mathrm{~nm}$, respectively (Fig. 1h). Meanwhile, an emission peak at $\sim 630 \mathrm{~nm}$ was observed. For Gd@C-dots made with $\mathrm{CA}$, on the other hand, the absorbance peak appears at $347 \mathrm{~nm}$, and the emission peak is centered at $449 \mathrm{~nm}$ (Fig. 1i).

These results indicate that two ultrasmall Gd@C-dots were successfully synthesized and they showed varied surface and optimal properties.

\section{Chemistry and radiochemistry}

Before in vivo therapy experiment, we aim to evaluate the cell uptake and clearance profile of the Gd@C-dots using radiolabeling approach. Therefore, radiometal chelators were introduced to the nanoparticles through amide bond formation using reported procedures [3436]. In brief, the carboxylic acid group in CA-Gd@C-dots was activated by EDC which was then conjugated with DOTA- $\mathrm{NH}_{2}$, leading to DOTA-CA-Gd@C-dots. Similarly, the amino group in pPD-Gd@C-dots reacted with DOTA-sNHS ester or NOTA-NCS leading to DOTApPD-Gd@C-dots or NOTA-pPD-Gd@C-dots ready for ${ }^{64} \mathrm{Cu}$ labeling. The chelator modified nanomaterials could all be efficiently labeled by ${ }^{64} \mathrm{Cu}$ with a yield ranging from 75\%-90\% (non-decay corrected).

\section{In vitro experiments \\ Cells and animals}

We investigated safety and efficacy of pPD-Gd@Cdots and CA-Gd@C-dots with H1299 cells, which is a human non-small cell lung cancer cell line. Both nanoparticles were non-toxic to H1299 cells (Fig. 2; Additional file 1: S2). To investigate the cell uptake difference between CA-Gd@C-dots and pPD-Gd@Cdots, we first incubated the ${ }^{64} \mathrm{Cu}$ labeled agents with $\mathrm{H} 1299$ cells. As shown in Fig. $3,{ }^{64} \mathrm{Cu}$-DOTACA-Gd@C-dots had lowest cell uptake at all time points tested, with the highest uptake observed being $5.6 \pm 0.2 \%$ of total activity at $240 \mathrm{~min}$. The cell uptake of ${ }^{64} \mathrm{Cu}$-DOTA-pPD-Gd@C-dots was significantly higher than ${ }^{64} \mathrm{Cu}$-DOTA-CA-Gd@C-dots, which was $1.9 \pm 1.3,4.5 \pm 1.3,17.7 \pm 9.1,25.9 \pm 4.6,32.4 \pm 13.6$, and $39.2 \pm 9.3 \%$ of total activity after $5,15,30,60$, 120 , and $240 \mathrm{~min}$ incubation, respectively. The cell uptake of ${ }^{64} \mathrm{Cu}$-NOTA-pPD-Gd@C-dots was the highest among three tracers, which was $3.6 \pm 3.9,4.9 \pm 2.4$, $29.1 \pm 8.8,37.2 \pm 4.5,48.7 \pm 2.6$, and $59.2 \pm 18.9$, after $5,15,30,60,120$, and $240 \mathrm{~min}$ incubation, respectively. In summary, ${ }^{64} \mathrm{Cu}$ labeled pPD-Gd@C-dots demonstrated more than 10 times higher cell uptake in vitro than ${ }^{64} \mathrm{Cu}$ labeled CA-Gd@C-dots. It is still unknown 


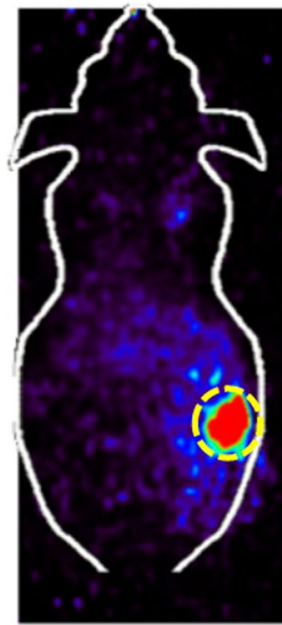

$1 \mathrm{~h}$

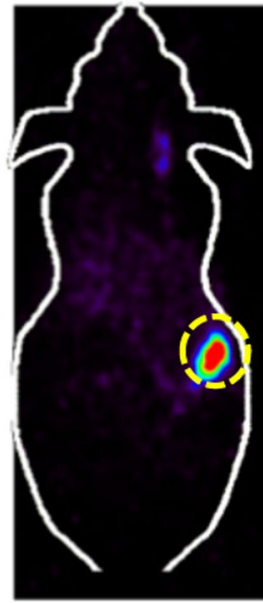

$1 \mathrm{~h}$

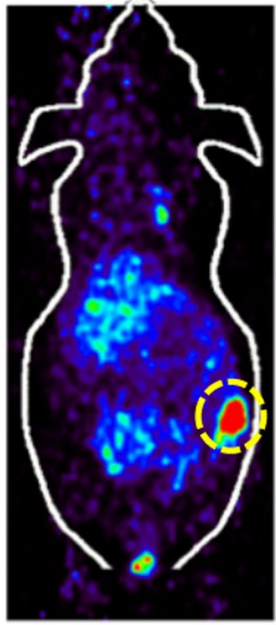

$4 \mathrm{~h}$

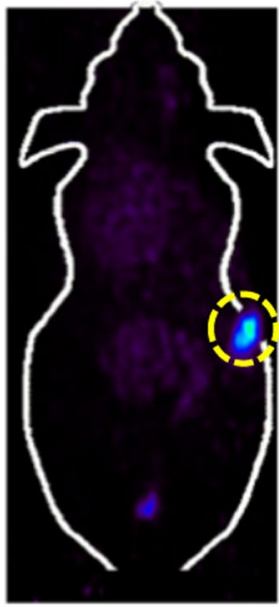

$4 \mathrm{~h}$

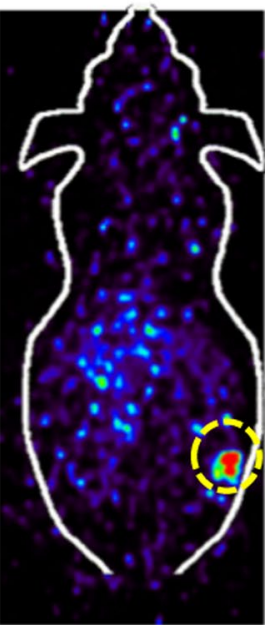

$24 \mathrm{~h}$

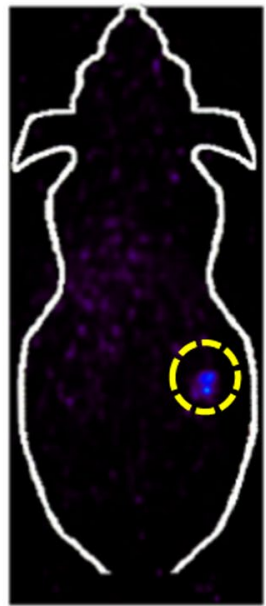

24h

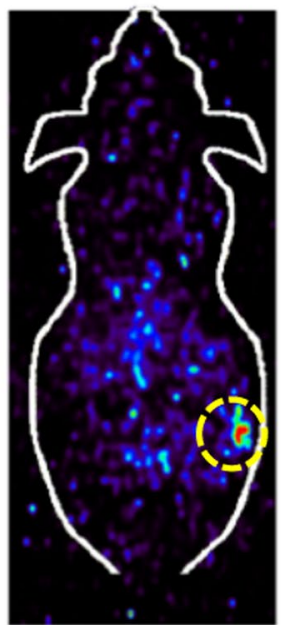

$48 \mathrm{~h}$

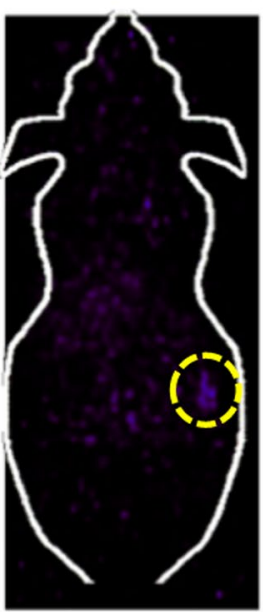

$300 \% 10 / 9$

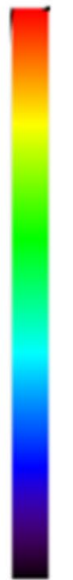

$48 \mathrm{~h}$

Fig. 6 Representative coronal small-animal PET images of mice bearing H1299 xenografts at 1, 4, 24, and 48 h after intratumoral injection of $0.89 \mathrm{MBq}$ of ${ }^{64} \mathrm{Cu}$-DOTA-CA-Gd@C-dots with scope bar100\%ID/g(top) and 300\%ID/g (bottom)

why ${ }^{64} \mathrm{Cu}$-DOTA-CA-Gd@C-dots showed higher uptake than ${ }^{64} \mathrm{Cu}-\mathrm{NOTA}-\mathrm{CA}-\mathrm{Gd} @ \mathrm{C}$-dots. Because only small percent of surface amino groups were modified, it is unlikely lead to significant changes on surface charges. The observed cell uptake difference may be attributed to the stability difference between $\mathrm{Cu}$ DOTA and Cu-NOTA complex, which still need to be confirmed in future studies. Because we did not observe significant difference between DOTA and NOTA modified pPD-Gd@C-dots, ${ }^{64} \mathrm{Cu}$-DOTA-pPDGd@C-dots was used for in vivo imaging studies to minimize potential impact from the chelator and made it comparable with ${ }^{64} \mathrm{Cu}$-DOTA-CA-Gd@C-dots.
In vivo small-animal PET studies

To evaluate whether the increased cell trapping/retention could also lead to significantly increased tumor retention in vivo, we studied the distribution of ${ }^{64} \mathrm{Cu}$-DOTA-CAGd@C-dots and ${ }^{64} \mathrm{Cu}-\mathrm{DOTA}-\mathrm{pPD}-\mathrm{Gd} @ \mathrm{C}$-dots using small-animal PET in mice bearing $\mathrm{H} 1299$ xenografts at $1,4,24$, and $48 \mathrm{~h}$ after Intratumoral injection. As shown in Figs. 4, 5, the tumor uptake of ${ }^{64} \mathrm{Cu}$-DOTA-pPDGd@C-dots showed minimal clearance within 24 h after injection $(386.4,352.9$, and $337.4 \% \mathrm{ID} / \mathrm{g}$ at $1,4,24 \mathrm{~h}$ p.i. respectively) which maintained at $149.3 \% \mathrm{ID} / \mathrm{g}$ at $48 \mathrm{~h}$ p.i.. Compared to tumor, the uptakes in kidneys, liver, muscle and bone were almost negligible (Figs. 4, 5). 

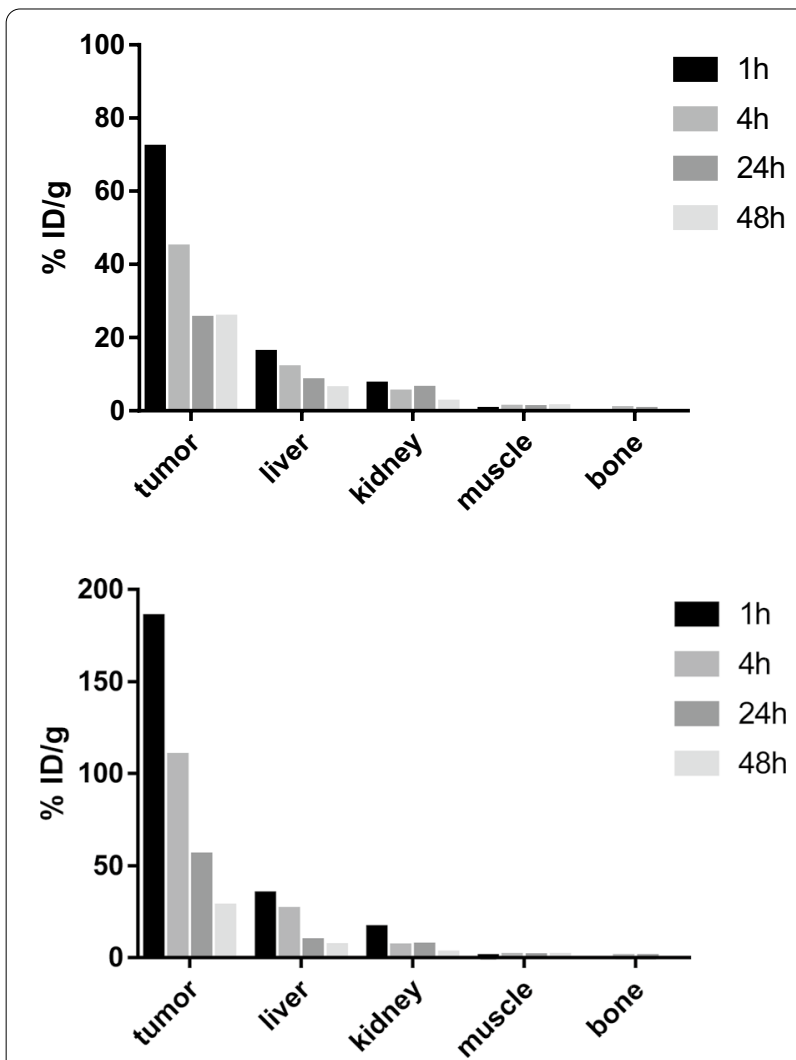

Fig. 7 Comparison of tumor, liver, kidney, muscle and bone uptake of ${ }^{64} \mathrm{Cu}$-DOTA-CA-Gd@C-dots at different time points $(1,4,24$, and $48 \mathrm{~h}$ ) after intratumoral injection

In comparison, ${ }^{64} \mathrm{Cu}$-DOTA-CA-Gd@C-dots demonstrated much faster clearance from the injection site. The tumor uptake in H1299 xenografts was 72.0, 44.7, 25.2 and $25.1 \% \mathrm{ID} / \mathrm{g}$ at $1,4,24$, and $48 \mathrm{~h}$ after intratumoral injection, respectively (Fig. 6). Different from ${ }^{64} \mathrm{Cu}$-DOTA-pPD-Gd@C-dots, we did observe ${ }^{64} \mathrm{Cu}$-DOTA-CA-Gd@C-dots demonstrated apparent uptake in kidneys and liver through the blood circulation. The kidney, liver, muscle and bone uptake were 15.9, 7.2, 0.3 , and $0.02 \% \mathrm{ID} / \mathrm{g}$ at $1 \mathrm{~h}$ after injection (Fig. 7). We like to point out that only one animal was used in each group, which showed large difference between two formulations. Although the obtained information is sufficient to guide us select the most promising formulation for therapy applications, additional numbers of animals would be needed if statistical analysis is required in future imaging analysis and comparison. In summary, ${ }^{64} \mathrm{Cu}$-DOTA-pPDGd@C-dots showed high and persistent tumor retention after injection, which makes it more suitable for in vivo treatment of tumors in following studies. Due to small particle size, both Figs. 4 and 6 shows that renal excretion is one of the main mechanisms for removing the radio drugs and nanoparticles from the body (especially after
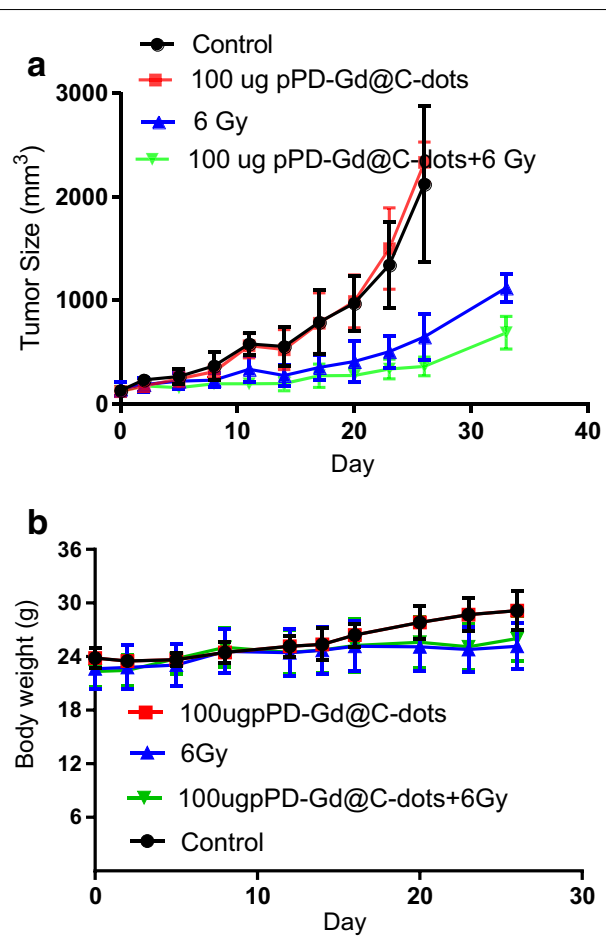

Fig. 8 X-Ray radiotherapy studies on H1299 tumor models. Animals received one session of treatment on Day 0 . a Tumor growth curves. Tumors were measured by monitoring tumor diameter changes at different time points. Compared to $X$-ray irradiation alone and pPD-Gd@C-dots injection only, significant tumor suppression was found with animals injected with pPD-Gd@C-dots and X-ray irradiation. b Body weight curves

$4 \mathrm{~h}$ ), This observation was also observed previously by other groups [37].

\section{X-ray radiotherapy studies}

Due to its high and persistent tumor retention, the following in vivo RT therapy studies were performed using pPD-Gd@C-dots in H1299 lung cancer model. In brief, the animals were randomly assigned to four groups: (1) No treatment, (2) $100 \mu \mathrm{g}$ pPD-Gd@C-dots, (3) 6 Gy radiation, and (4) $100 \mu \mathrm{g}$ pPD-Gd@C-dots + 6 Gy radiation. Our first set of experiment include single dose treatment and the results were shown in Fig. 8a. No treatment and $100 \mu \mathrm{g}$ pPD-Gd@C-dots group showed fast tumor progression, and the tumor reached an end point within 28 days. In contrast, 6 Gy irradiation clearly demonstrated certain inhibitory effect on tumor growth, and the treatment efficacy was further improved once pPD-Gd@C-dots were used as a combination therapy. No significant weight drop was found in all treatment groups (Fig. 8b).

According to the imaging study,pPD-Gd@Cdots have high tumor retention overtime. We then evaluated 

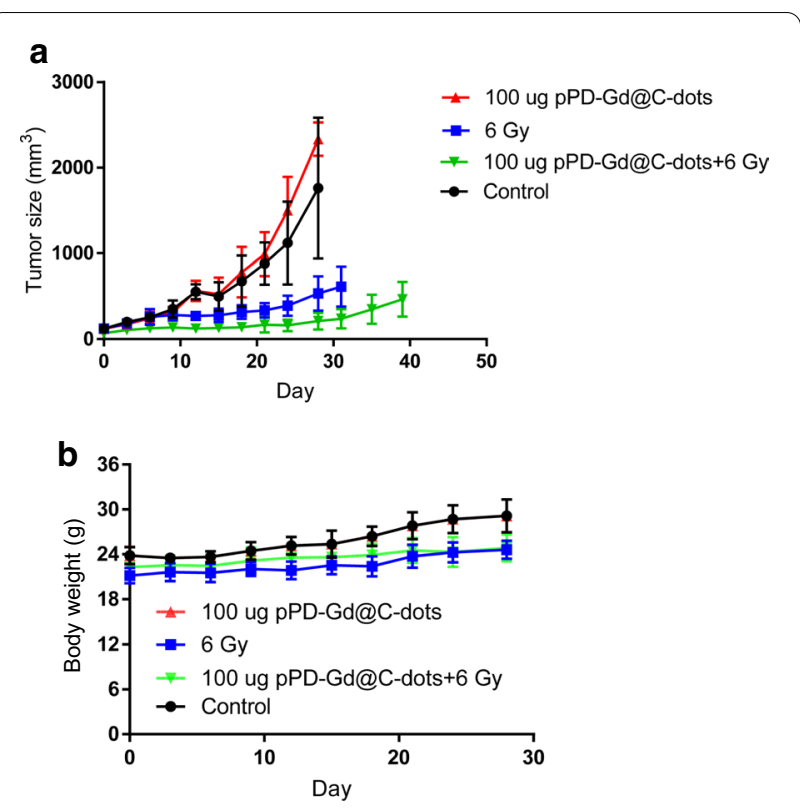

Fig. 9 X-Ray radiotherapy studies on $\mathrm{H} 1299$ tumor models. Animals received two doses of treatment on Days 0 and Day 3. a Tumor growth curves. Tumors were measured by monitoring tumor dimension changes at different time points. Compared to X-ray irradiation alone and pPD-Gd@C-dots injection only, significant tumor suppression was found with animals treated with pPD-Gd@C-dots plus irradiation.b Body weight curves

effective radiosensitizer. We also like to point out that even though we did not observe significant toxicity of Gd@C-dots in the current and previous studies [40-42], potential long-term side effects of these carbon dots still need to be evaluated further in follow-up studies, especially at high doses. The obtained information will be critical to clinical translation of these formulations in the future.

\section{Supplementary Information}

The online version contains supplementary material available at https://doi. org/10.1186/s12951-021-01018-9.

Additional file 1: Figure S1. Photos of pPD-Gd@C-dots (left) and CAGd@C-dots (right) in water. The solutions appeared dark red (pPD-Gd@Cdots) and brown (CA-Gd@C-dots), respectively. Figure S2. Cytotoxicity of pPD-Gd@C-dots and CA-Gd@C-dots, tested on H1299 cells using ATP bioluminescence assay. Figure S3. FT-IR spectra of Gd-CA@C-dots and pPD-Gd@C-dots, recorded on a Thermo Nicolet ${ }^{\mathrm{TM}}$ is $^{\mathrm{TM}} 10$ FTIR Spectrometer. Figure S4. Hydrodynamic sizes of Gd-CA@C-dots and pPD-Gd@Cdots, measured on a Malvern Zetasizer Nano ZS system. The average diameters are 2.13 and 2.05, respectively.

\section{Acknowledgements}

This work was supported by UNC Department of Radiology, Biomedical Research Imaging Center, and Lineberger Comprehensive Cancer Center (Z.L.); the science foundation of Guangdong Second Provincial General hospital (Grant Number: YN2018-001), and the Science and Technology Program of Guangzhou, China (Grant Number: 201804010448) (X.M.).

\section{Disclosure}

A patent application has been filed based on the C-dot nanoparticle.

whether introducing a second 6 Gy irradiation (without re-injecting nanoparticle) could further improve treatment efficacy. As shown in Fig. 9, the tumor inhibition efficacy was further improved in the combination treatment group. No apparent body weight loss was observed. As shown in Figs. 8a and 9a, the tumor size increased significantly at late time points which may indicate the recurrence of the disease $[38,39]$. In clinic, multi-session fractionated radiotherapy with a cumulative dose of $\sim 60$ Gy could be applied to lung cancer management. Due significant normal tissue toxicity, multi-session radiotherapy is not feasible for small animals. Whether the Cdots could achieve better therapeutic effect in clinic settings are still need to be investigated.

\section{Conclusion}

Gd@C-dots have demonstrated the great potential to be used as radiation sensitizer due to their high stability, minimal metal leakage, and remarkable radiosensitizing effects. In this report, we demonstrated that PET offers a simple and straightforward way to study nanoparticle retention in vivo. The surface functional group could have significant impact on nanoparticle uptake and retention. The selected pPD-Gd@C-dots hold great potential as an

\section{Authors' contributions}

$J X, G J$ and ZL conceptualized and supervised the project. XM, CL and TZ. Synthesized particles, performed imaging studies. XM and JC carried out therapy studies. XM, CL and TZ wrote the manuscript with contribution from other authors. All authors read and approved the final manuscript.

Received: 24 June 2021 Accepted: 29 August 2021

Published online: 22 September 2021

\section{References}

1. Abshire D, Lang MK. The evolution of radiation therapy in treating cancer. Semin Oncol Nurs. 2018;34:151-7.

2. Baskar R, Lee KA, Yeo R, Yeoh KW. Cancer and radiation therapy: current advances and future directions. Int J Med Sci. 2012;9:193-9.

3. Sampath S. Treatment: radiation therapy. Cancer Treat Res. 2016;170:105-18.

4. De Ruysscher D, Niedermann G, Burnet NG, Siva S, Lee AWM, Hegi-Johnson F. Radiotherapy toxicity. Nat Rev Dis Primers. 2019;5:13.

5. Straub JM, New J, Hamilton CD, Lominska C, Shnayder Y, Thomas SM. Radiation-induced fibrosis: mechanisms and implications for therapy. J Cancer Res Clin Oncol. 2015;141:1985-94.

6. Goldstein M, Kastan MB. The DNA damage response: implications for tumor responses to radiation and chemotherapy. Annu Rev Med. 2015;66:129-43.

7. Jarosz-Biej M, Smolarczyk R, Cichon T, Kulach N. Tumor microenvironment as a "Game Changer" in cancer radiotherapy. Int J Mol Sci. 2019;20:3212.

8. Vilotte F, Jumeau R, Bourhis J. High Z nanoparticles and radiotherapy: a critical view. Lancet Oncol. 2019;20:e557. 
9. Li Y, Yun KH, Lee H, Goh SH, Suh YG, Choi Y. Porous platinum nanoparticles as a high-Z and oxygen generating nanozyme for enhanced radiotherapy in vivo. Biomaterials. 2019;197:12-9.

10. Subiel A, Ashmore R, Schettino G. Standards and methodologies for characterizing radiobiological impact of high-Z nanoparticles. Theranostics. 2016;6:1651-71.

11. Dufort S, Le Duc G, Salome M, Bentivegna V, Sancey L, Brauer-Krisch E, Requardt H, Lux F, Coll JL, Perriat P, et al. The high radiosensitizing efficiency of a trace of gadolinium-based nanoparticles in tumors. Sci Rep. 2016;6:29678

12. Meidanchi A, Akhavan O, Khoei S, Shokri AA, Hajikarimi Z, Khansari N. ZnFe2O4 nanoparticles as radiosensitizers in radiotherapy of human prostate cancer cells. Mater Sci Eng C Mater Biol Appl. 2015;46:394-9.

13. Rima W, Sancey L, Aloy MT, Armandy E, Alcantara GB, Epicier T, Malchere A, Joly-Pottuz L, Mowat P, Lux F, et al. Internalization pathways into cancer cells of gadolinium-based radiosensitizing nanoparticles. Biomaterials. 2013;34:181-95

14. Deng J, Xu S, Hu W, Xun X, Zheng L, Su M. Tumor targeted, stealthy and degradable bismuth nanoparticles for enhanced $X$-ray radiation therapy of breast cancer. Biomaterials. 2018:154:24-33.

15. Rajaee A, Wang S, Zhao L, Wang D, Liu Y, Wang J, Ying K. Multifunction bismuth gadolinium oxide nanoparticles as radiosensitizer in radiation therapy and imaging. Phys Med Biol. 2019;64:195007.

16. Wu B, Lu ST, Yu H, Liao RF, Li H, Lucie Zafitatsimo BV, Li YS, Zhang Y, Zhu $X \mathrm{~L}$, Liu HG, et al. Gadolinium-chelate functionalized bismuth nanotheranostic agent for in vivo MRI/CT/PAl imaging-guided photothermal cancer therapy. Biomaterials. 2018;159:37-47.

17. Yong Y, Cheng X, Bao T, Zu M, Yan L, Yin W, Ge C, Wang D, Gu Z, Zhao Y. Tungsten sulfide quantum dots as multifunctional nanotheranostics for in vivo dual-modal image-guided photothermal/radiotherapy synergistic therapy. ACS Nano. 2015;9:12451-63.

18. Wang H, Dong W, Zhao Q, Lu K, Guo X, Liu H, Wu Z, Li S. Synthesis of N-(6[(18)F]Fluoropyridin-3-yl)glycine as a potential renal PET agent. Nucl Med Biol. 2019;76-77:21-7.

19. Zang Y, Gong L, Mei L, Gu Z, Wang Q. Bi2WO6 semiconductor nanoplates for tumor radiosensitization through high-Z effects and radiocatalysis. ACS Appl Mater Interfaces. 2019;11:18942-52.

20. Bonvalot S, Rutkowski PL, Thariat J, Carrere S, Ducassou A, Sunyach MP, Agoston P, Hong A, Mervoyer A, Rastrelli M, et al. NBTXR3, a first-in-class radioenhancer hafnium oxide nanoparticle, plus radiotherapy versus radiotherapy alone in patients with locally advanced soft-tissue sarcoma (Act.In.Sarc): a multicentre, phase 2-3, randomised, controlled trial. Lancet Oncol. 2019;20:1148-59.

21. Khalladi N, Thariat J. Doubling complete histological response in sarcomas with radiation therapy using nanoparticles (Hafnium oxide, NBTXR3), a phase III trial. Bull Cancer. 2019;106:1070-2.

22. Chen Y, Zhong H, Wang J, Wan X, Li Y, Pan W, Li N, Tang B. Catalaselike metal-organic framework nanoparticles to enhance radiotherapy in hypoxic cancer and prevent cancer recurrence. Chem Sci. 2019;10:5773-8

23. Huang Q, Zhang S, Zhang H, Han Y, Liu H, Ren F, Sun Q, Li Z, Gao M. Boosting the radiosensitizing and photothermal performance of Cu2-xSe nanocrystals for synergetic radiophotothermal therapy of orthotopic breast cancer. ACS Nano. 2019;13:1342-53.

24. Bai L, Jiang F, Wang $R$, Lee C, Wang H, Zhang W, Jiang W, Li D, Ji B, Li Z, et al. Ultrathin gold nanowires to enhance radiation therapy. J Nanobiotechnology. 2020;18:131.

25. Briggs A, Corde S, Oktaria S, Brown R, Rosenfeld A, Lerch M, Konstantinov K, Tehei M. Cerium oxide nanoparticles: influence of the high-Z component revealed on radioresistant $9 \mathrm{~L}$ cell survival under $\mathrm{X}$-ray irradiation. Nanomedicine. 2013;9:1098-105.
26. Zhu WZ, Wen YC, Lin SY, Chen TC, Chen HW. Anti-influenza protective efficacy of a H6 virus-like particle in chickens. Vaccines (Basel). 2020;8:465.

27. Sieber S, Grossen P, Detampel P, Siegfried S, Witzigmann D, Huwyler J. Zebrafish as an early stage screening tool to study the systemic circulation of nanoparticulate drug delivery systems in vivo. J Control Release. 2017;264:180-91.

28. Liu L, Law WC, Yong KT, Roy I, Ding H, Erogbogbo F, Zhang X, Prasad PN. Multimodal imaging probes based on Gd-DOTA conjugated quantum dot nanomicelles. Analyst. 2011;136:1881-6.

29. Fakhry-Darian D, Patel NH, Khan S, Barwick T, Svensson W, Khan S, Perry RJ, Malhotra P, Carswell CJ, Nijran KS, Win Z. Optimisation and usefulness of quantitative analysis of (18)F-florbetapir PET. Br J Radiol. 2019;92:20181020.

30. Ma X, Wang M, Wang H, Zhang T, Wu Z, Sutton MV, Popik VV, Jiang G, Li Z. Development of bispecific NT-PSMA heterodimer for prostate cancer imaging: a potential approach to address tumor heterogeneity. Bioconjug Chem. 2019;30:1314-22.

31. Chen H, Wang GD, Tang W, Todd T, Zhen Z, Tsang C, Hekmatyar K, Cowger T, Hubbard R, Zhang W, et al. Gd-encapsulated carbonaceous dots with efficient renal clearance for magnetic resonance imaging. Adv Mater. 2014;26:6761-6.

32. Wu Y, Li H, Yan Y, Wang K, Cheng Y, Li Y, Zhu X, Xie J, Sun X. Affibodymodified Gd@C-Dots with efficient renal clearance for enhanced MRI of EGFR expression in non-small-cell lung cancer. Int J Nanomed. 2020;15:4691-703.

33. Wu Y, Yan Y, Gao X, Yang L, Li Y, Guo X, Xie J, Wang K, Sun X. Gd-encapsulated carbonaceous dots for accurate characterization of tumor vessel permeability in magnetic resonance imaging. Nanomedicine. 2019;21:102074.

34. Yang HM, Park CW, Park S, Kim JD. Cross-linked magnetic nanoparticles with a biocompatible amide bond for cancer-targeted dual optical/magnetic resonance imaging. Colloids Surf B Biointerfaces. 2018;161:183-91.

35. Rana S, Bharti A, Singh S, Bhatnagar A, Prabhakar N. Gold-silver core-shell nanoparticle-based impedimetric immunosensor for detection of iron homeostasis biomarker hepcidin. Mikrochim Acta. 2020;187:626.

36. Kollner S, Dunnhaupt S, Waldner C, Hauptstein S, Pereira de Sousa I, Bernkop-Schnurch A. Mucus permeating thiomer nanoparticles. Eur J Pharm Biopharm. 2015;97:265-72.

37. Fazaeli Y, Akhavan O, Rahighi R, Aboudzadeh MR, Karimi E, Afarideh H. In vivo SPECT imaging of tumors by 198,199Au-labeled graphene oxide nanostructures. Mater Sci Eng C Mater Biol Appl. 2014;45:196-204.

38. Yang K, Zhang S, Zhang G, Sun X, Lee ST, Liu Z. Graphene in mice: ultrahigh in vivo tumor uptake and efficient photothermal therapy. Nano Lett. 2010;10:3318-23.

39. Akhavan O, Ghaderi E. Graphene nanomesh promises extremely efficient in vivo photothermal therapy. Small. 2013;9:3593-601.

40. Chen H, Qiu Y, Ding D, Lin H, Sun W, Wang GD, Huang W, Zhang W, Lee D, Liu G, et al. Gadolinium-encapsulated graphene carbon nanotheranostics for imaging-guided photodynamic therapy. Adv Mater. 2018;2018:e1802748.

41. Lee C, Liu X, Zhang W, Duncan MA, Jiang F, Kim C, Yan X, Teng Y, Wang H, Jiang W, et al. Ultrasmall Gd@Cdots as a radiosensitizing agent for nonsmall cell lung cancer. Nanoscale. 2021;13:9252-63.

42. Chen H, Wang GD, Sun X, Todd T, Zhang F, Xie J, Shen B. Mesoporous silica as nanoreactors to prepare $\mathrm{Gd}$-encapsulated carbon dots of controllable sizes and magnetic properties. Adv Func Mater. 2016;26:3973-82.

\section{Publisher's Note}

Springer Nature remains neutral with regard to jurisdictional claims in published maps and institutional affiliations. 